\title{
Level I Evidence Should not be Equated to Standard of Care for Brain Metastases
}

Keywords: Brain metastases, Stereotactic radiosurgery, Whole-brain radiation therapy

doi:10.1017/cjn.2020.250

Can J Neurol Sci. 2021; 48: 155-156

In this issue, O'Halloran et al. ${ }^{1}$ have written a comprehensive review of current and upcoming management options for brain metastases. The goal of this editorial is to provide a pragmatic neurosurgical perspective on this matter.

We usher into an unprecedented era in oncology. Molecular diagnosis has led to a revolution in the management of malignancies that are most frequently responsible for the development of brain metastases, such as non-small cell lung cancer, melanoma, and breast cancer. Immune checkpoint inhibitors and targetspecific tyrosine kinase inhibitors have been demonstrated to provide significant improvement in the overall survival of such patients. The proportion of patients who will develop and live for a prolonged period of time with brain metastases is expected to increase in the coming years. Consequently, therapies used to manage these tumors have to be safe and be able to be delivered quickly without disrupting the schedule of systemic therapy.

Unfortunately, the improvements in systemic managements have not been able to provide any significant benefit for brain therapy. The blood-brain barrier penetration of newer agents has improved, but they still cannot be expected to produce durable response in the brain, and as such, radiation therapy remains the mainstay of brain metastases management. However, radiation therapy has seen its own share of refinements over the years. In the early eras of cancer management, the onset of brain metastases was indicative of a certain doom for afflicted patients who were usually offered palliative management. Lack of appropriate imaging to identify small brain metastases and technical limitations of older radiation delivery devices led to the establishment of whole-brain radiation therapy (WBRT) as the de facto standard of care for brain metastases. Randomized studies showed that WBRT led to improvement in symptoms over supportive care alone but survival remained short ${ }^{2}$. For single brain metastases, surgical resection followed by WBRT was found superior to WBRT alone or resection alone ${ }^{3,4}$. However, over time it became apparent that the few patients who lived long enough developed widespread leukoencephalopathy secondary to WBRT and had a significant risk of associated cognitive disorders 5 . In a recent study, hippocampal avoidance (HA) WBRT combined with memantine was demonstrated to mitigate this risk compared to standard WBRT ${ }^{6}$. However, the risk of cognitive failure at 3 months was still more than $50 \%$ in both groups. Consequently, in most centers treating brain metastases patients, WBRT has fallen out of favor and is mostly used for the management of miliary metastatic disease, leptomeningeal dissemination, and patients with very poor performance status.

For the majority of neuro-oncology indications, newer radiation techniques such as intensity modulated radiation therapy
(IMRT) and stereotactic radiosurgery (SRS) have now become the standard modalities to spare normal tissues from the potential deleterious effects of radiation. The newer SRS devices, such as the Gamma Knife Perfexion ${ }^{\circledR} /$ Icon $^{\circledR}$ (Elekta AB) and dedicated linear accelerators (Accuray CyberKnife ${ }^{\circledR}$, Brainlab Novalis ${ }^{\circledR}$ ), are able to deliver lethal radiation doses to brain metastases and provide a sharp dose falloff to the surrounding normal brain parenchyma. SRS has multiple practical advantages for brain metastases patients. It is usually delivered over a single day, minimizing the need for patient travel to the treating facility, and can be easily scheduled between systemic therapy sessions, so that no interruption is required. Despite the lack of true level I studies, SRS has been demonstrated to be an effective and well-tolerated technique in multiple publications over the past years, with high tumor control rates and minimal risks of adverse effects. For patients with a limited number of brain metastases (one to four), multiple randomized studies have compared SRS alone to SRS combined with WBRT, either as primary treatment or in adjuvant setting after resection ${ }^{7-11}$. All those studies came to similar conclusions. The omission of WBRT did not affect overall survival and local control of treated tumors but led to increased risk of developing new remote brain metastases (that can be managed by additional SRS sessions as needed). Patients included in the WBRT groups showed worsening of cognitive function and health-related quality of life compared to patients treated with SRS alone. The JLGK0901 study, a large nonrandomized prospective study of SRS alone for up to 10 brain metastases, has demonstrated similar survival for patients who had 2-4 tumors compared to those with 5-10 tumors, and that total tumor volume, not absolute tumor number, was better correlated with overall survival $^{12}$. A further analysis of all three subgroups of that study demonstrated that the rates of post-SRS complications and cognitive deterioration were not affected by the number of treated tumors and remained very low over the follow-up period ${ }^{13}$.

The Canadian Cancer Trials Group is currently running a trial that is comparing SRS alone to HA-WBRT and memantine for patients with 5 to 15 brain metastases (CCTG CE7). While the intent to generate the best level of evidence-based medicine has to be commended, the results of this study will likely be similar to those of prior trials involving patients with lower number of brain metastases. If all other factors are similar, it is unlikely that a patient with 12 metastases will fare worse than one with only 9, for example. And after this study is done, what will the next steps be? At some point, the absolute number of metastases is arbitrarily set and has to be taken into consideration to guide management as only one of multiple factors defining patient 
condition, along with performance index, age, systemic disease status, and molecular subtypes ${ }^{14}$.

In most practice guidelines for brain metastases (as reported by O'Halloran et al.), WBRT remains an option based on level I evidence, while SRS is supported by level III evidence only ${ }^{15,16}$. This can be misleading for readers who are not familiar with the current literature on this topic. Brain metastases patients with good performance status can expect to have prolonged survival with the newer systemic therapies available for their primary disease. For those patients, SRS should be considered as the standard of care management to provide tumor control and preservation cognition and quality of life, irrespective of evidence level.

\section{Disclosure}

Dr. Mathieu has nothing to disclose.

David Mathieu

Division of Neurosurgery, Department of Surgery, Université de Sherbrooke, Centre de recherche du CHUS, Sherbrooke, QC, Canada

Correspondence to: David Mathieu, Service de neurochirurgie, Centre Hospitalier Universitaire de Sherbrooke, 3001, $12^{\mathrm{e}}$ avenue Nord, Sherbrooke, QC, Canada, J1H5N4.Email: david.mathieu@ usherbrooke.ca

\section{REFERENCES}

1. O'Halloran PJ, Gutierrez E, Kalyvas A, et al. Brain metastases: a modern multidisciplinary approach. Can J Neurol Sci. 2021;48:189-97. doi: 10.1017/cjn.2020.224.

2. Borgelt B, Gelber R, Kramer S, et al. The palliation of brain metastases: final results of the first two studies by the Radiation Therapy Oncology Group. Int J Radiat Oncol Biol Phys. 1980;6(1):1-9. doi: 10.1016/0360-3016(80)90195-9.

3. Patchell RA, Tibbs PA, Walsh JW, et al. A randomized trial of surgery in the treatment of single metastases to the brain. N Engl J Med. 1990;322(8):494-500.

4. Patchell RA, Tibbs PA, Regine WF, et al. Postoperative radiotherapy in the treatment of single metastases to the brain: a randomized trial. JAMA. 1998;280(17):1485-89.

5. DeAngelis LM, Delattre JY, Posner JB. Radiation-induced dementia in patients cured of brain metastases. Neurology. 1989;39(6):789-96.

6. Brown PD, Gondi V, Pugh S, et al. Hippocampal avoidance during whole-brain radiotherapy plus memantine for patients with brain metastases: phase III trial NRG oncology CC001. J Clin Oncol. 2020;38(10):1019-29. doi: 10.1200/JCO.19.02767.

7. Aoyama H, Shirato H, Tago M, et al. Stereotactic radiosurgery plus whole-brain radiation therapy vs stereotactic radiosurgery alone for treatment of brain metastases: a randomized controlled trial. JAMA. 2006;295(21):2483-91. doi: 10.1001/jama.295.21. 2483.

8. Chang EL, Wefel JS, Hess KR, et al. Neurocognition in patients with brain metastases treated with radiosurgery or radiosurgery plus whole-brain irradiation: a randomised controlled trial. Lancet Oncol. 2009;10(11):1037-44. doi: 10.1016/S1470-2045(09) 70263-3.

9. Kocher M, Soffietti R, Abacioğlu U, et al. Adjuvant whole-brain radiotherapy versus observation after radiosurgery or surgical resection of one to three cerebral metastases: results of the EORTC 22952-26001 study. J Clin Oncol. 2011;29(2):134-41. doi: $10.1200 / \mathrm{JCO} .2010 .30 .1655$.

10. Brown PD, Jaeckle K, Ballman KV, et al. Effect of radiosurgery alone vs radiosurgery with whole brain radiation therapy on cognitive function in patients with 1 to 3 brain metastases: a randomized clinical trial. JAMA. 2016;316(4):401-09. doi: 10. 1001/jama.2016.9839.

11. Brown PD, Ballman KV, Cerhan JH, et al. Postoperative stereotactic radiosurgery compared with whole brain radiotherapy for resected metastatic brain disease (NCCTG N107C/CEC3): a multicentre, randomised, controlled, phase 3 trial. Lancet Oncol. 2017;18(8):1049-60. doi: 10.1016/S1470-2045(17)30441-2.

12. Yamamoto M, Serizawa T, Shuto T, et al. Stereotactic radiosurgery for patients with multiple brain metastases (JLGK0901): a multiinstitutional prospective observational study. Lancet Oncol. 2014;15(4):387-95. doi: 10.1016/S1470-2045(14)70061-0.

13. Yamamoto M, Serizawa T, Higuchi Y, et al. A multi-institutional prospective observational study of stereotactic radiosurgery for patients with multiple brain metastases (JLGK0901 Study Update): irradiation-related complications and long-term maintenance of mini-mental state examination scores. Int J Radiat Oncol Biol Phys. 2017;99(1):31-40. doi: 10.1016/j.ijrobp.2017.04.037.

14. Sperduto PW, Mesko S, Li J, et al. Survival in patients with brain metastases: summary report on the updated diagnosis-specific graded prognostic assessment and definition of the eligibility quotient. J Clin Oncol. 2020;38:3773-84. doi: 10.1200/JCO.20. 01255 .

15. Gaspar LE, Prabhu RS, Hdeib A, et al. Congress of neurological surgeons systematic review and evidence-based guidelines on the role of whole brain radiation therapy in adults with newly diagnosed metastatic brain tumors. Neurosurgery. 2019;84(3): E159-E162. doi: 10.1093/neuros/nyy541.

16. Graber JJ, Cobbs CS, Olson JJ. Congress of neurological surgeons systematic review and evidence-based guidelines on the use of stereotactic radiosurgery in the treatment of adults with metastatic brain tumors. Neurosurgery. 2019;84(3):E168-E170. doi: 10. 1093/neuros/nyy543. 